\title{
Parâmetros genotípicos, fenotípicos e ambientais para caracteres morfológicos e agronômicos em abóbora
}

\author{
Arie F Blank; Thássia B Silva; Maurício L Matos; José LS Carvalho Filho; Renata Silva-Mann
}

${ }^{1}$ UFS-DEA, Av. Marechal Rondon s/n, 49100-000 São Cristóvão-SE; afblank@ufs.br; thassia1984@yahoo.com.br; mlmatoz@yahoo. com.br; jlsandes2000@yahoo.com.br; renatamann@hotmail.com

\section{RESUMO}

A abóbora é uma hortaliça que apresenta grande importância na agricultura familiar no nordeste brasileiro. Muitas comunidades têm encontrado dificuldades na comercialização de todos os frutos produzidos, em função da grande variabilidade fenotípica, que nem sempre atende às exigências do mercado. $\mathrm{O}$ estudo de parâmetros genéticos é importante para fornecer informações na implantação e condução de um programa de melhoramento. Desta forma, o objetivo do trabalho foi estimar parâmetros genéticos, fenotípicos e ambientais para caracteres morfológicos e agronômicos em abóbora. Foram estudados sete genótipos de abóbora para os anos agrícolas de 2008 e 2009, considerando como aleatório o efeito dos anos e fixo o efeito dos genótipos. As variáveis analisadas foram comprimento e largura do fruto, espessura da polpa na região do pedúnculo, do meio e da parte inferior do fruto, altura e largura da cavidade interna do fruto, rendimento de frutos, número de sementes por fruto e massa de 100 sementes. A variável que apresentou os maiores coeficientes de variação genética (25,10 e 41,32 nos dois anos agrícolas, respectivamente) foi a espessura da polpa no pedúnculo. A herdabilidade foi acima de $90 \%$ para as variáveis comprimento dos frutos (98 e 97\%), largura dos frutos (98 e 95\%), espessura da polpa na região do pedúnculo (98 e 96\%), e altura da cavidade interna do fruto (99 e 97\%), nos dois anos agrícolas avaliados. Para a relação $\mathrm{CV}_{\mathrm{g}} / \mathrm{CV}_{\mathrm{e}}$, foram encontrados valores maiores que 1,0 para os caracteres comprimento e largura do fruto, espessura da polpa na região do pedúnculo e do meio, largura e altura da cavidade interna dos frutos, rendimento de frutos, número de sementes por fruto e massa fresca de 100 sementes. Na análise conjunta das médias, os genótipos se comportaram diferentemente em relação aos caracteres avaliados.

Palavras-chave: Cucurbita moschata, melhoramento genético, variabilidade, variância, herdabilidade.

\begin{abstract}
Genotypic, phenotypic and environmental parameters for morphological and agronomic characters in pumpkin

Pumpkin is a vegetable that has a great importance on family farm in northeastern Brazil. Many communities have found difficulties in the commercialization of all the produced fruits, due to the large phenotypic variability, which does not always attend the market requirements. The study of genetic parameters is important to provide information on the deployment and conduction of breeding programs. Thus, the aim of this study was to estimate genetic, phenotypic and environmental parameters for morphological and agronomic characters in pumpkin. Seven pumpkin genotypes were studied in the crop years 2008 and 2009, considering as random the effect of years and as fixed the effect of populations. The analyzed variables were fruit length and width, pulp thickness in the peduncle, the middle and the lower region of the fruit, height and width of the fruit internal cavity, fruit yield, number of seeds per fruit and fresh weight of 100 seeds. Pulp thickness in the peduncle presented the highest coefficients of genetic variation, 25.10 and 41.32 in the two evaluated agricultural years, respectively. Heritability was above $90 \%$ for the variables fruit length (98 and 97\%), fruit width (98 and 95\%), pulp thickness in the peduncle and in the middle region of the fruit (98 and 96\%), and height of the fruit internal cavity (99 and 97\%), in the two evaluated agricultural years. For the $\mathrm{CV}_{\mathrm{g}} / \mathrm{CV}_{\mathrm{e}}$ ratio, the values were greater than 1.0 for the characters fruit length and width, pulp thickness in the peduncle and in the middle region of the fruit, width and height of the fruit internal cavity, fruit yield, number of seeds per fruit, and fresh weight of 100 seeds. In the joint analysis of means, the genotypes showed different behavior in relation to the evaluated characters.
\end{abstract}

Keywords: Cucurbita moschata, breeding, variability, variance, herdability.

\section{(Recebido para publicação em 17 de novembro de 2011; aceito em 18 de janeiro de 2013) (Received on November 17, 2011; accepted on January 18, 2013)}

\begin{abstract}
$\mathrm{O}$ gênero Cucurbita, nativo das Américas, é constituído por 15 espécies, sendo a abóbora (Cucurbita moschata) e a moranga (Cucurbita maxima) as principais espécies cultivadas. A grande variabilidade genética das abóboras é de suma importância para a alimentação humana tanto pela versatilidade culinária (Silva et al., 2006) quanto pela riqueza em
\end{abstract}

caroteno, ferro, cálcio, magnésio, potássio e vitaminas A, B e C (Filgueira, 2008). Um quilo é constituído por $13 \%$ de fibras e $96 \%$ de água, composto por 40 calorias, $280 \mathrm{mg}$ de vitamina A, $700 \mathrm{mg}$ de vitamina B5, $100 \mathrm{mg}$ de vitamina $\mathrm{B} 2,55 \mathrm{mg}$ de vitamina B, além de sais (Luengo et al., 2000). No Brasil, a diversidade genética de abóboras concentra-se especialmente na região Nordeste (Assis et al., 2006). No estado de Sergipe, especificamente no município de Simão Dias, a produção de abóbora supre o estado e outros como Pernambuco, Alagoas e Bahia. No entanto na escolha dos frutos para comercialização, muitos são deixados na propriedade por não atender ao formato exigido pelo consumidor. O material cultivado na região apresenta-se bem 
adaptado às condições edafoclimáticas, podendo ser usado em programas de melhoramento genético.

A eficiência do melhoramento depende do conhecimento do controle genético dos caracteres a serem melhorados (Basso et al., 2009). O conhecimento de parâmetros genéticos como herdabilidade $\left(\mathrm{h}^{2}\right)$, componente de variabilidade genotípica $(\mathrm{Vg})$ e índice de variação (razão $\mathrm{CV}_{\mathrm{g}} / \mathrm{CV}_{\mathrm{e}}$ ), é de grande importância para o melhorista, uma vez que orienta a escolha do método de melhoramento mais adequado à cultura, maximizando ganhos com seleção (Cruz \& Regazzi, 2004).

A variabilidade observada para um determinado caráter nem sempre é transmitida de geração para geração, evidenciando que o efeito do ambiente é a principal causa de variação (Redig, 2007). A seleção é mais efetiva quando age sobre caracteres de alta herdabilidade e que tenham associação com a produção ou outro caráter de importância econômica (Gabriel, 2006).

A herdabilidade mede a proporção da variação fenotípica na população atribuída à causa genética (Cruz, 2005). Essa proporção pode ser alterada pelo efeito do ambiente. O coeficiente de herdabilidade é considerado como um dos parâmetros genéticos mais importantes, pois indica a proporção da variância fenotípica atribuída ao efeito médio dos genes e também seu papel preditivo, por expressar a confiabilidade do valor fenotípico como guia para selecionar um valor genético (Ruiz et al., 2004).

Desta forma, visando aumentar a eficiência na seleção em populações de abóbora, estimaram-se os parâmetros genéticos, fenotípicos e ambientais para caracteres morfológicos e agronômicos.

\section{MATERIAL E MÉTODOS}

Foram conduzidos experimentos em duas épocas, em campo da UFS, no município de São Cristóvão-SE. O primeiro experimento foi conduzido nos meses de janeiro a maio de 2008 . O segundo experimento nos meses de julho a novembro de 2009. Dentre os descritores morfoagronômicos propostos pelo Serviço Nacional de Proteção de
Cultivares (SNPC) para as espécies do gênero Cucurbita foram selecionados e adaptados 10 para a caracterização dos frutos: 1) rendimento de frutos ( $\left.\mathrm{t} \mathrm{ha}^{-1}\right)$ (massa total dos frutos da parcela útil); 2) comprimento e largura do fruto $(\mathrm{cm})$ (medido com régua graduada, obtendo as medidas longitudinal e transversal); 3) altura e largura da cavidade interna do fruto $(\mathrm{cm})$ (medido com régua graduada); 4) espessura da polpa na região do pedúnculo, do meio e da parte inferior do fruto $(\mathrm{cm})$ (medido com régua graduada); 5) número de sementes por fruto e 6) massa fresca de 100 sementes $(\mathrm{g})$.

Foram utilizadas quatro cultivares comerciais de abóbora (Itapuã, Menina Rajada, Mini Paulista e Nova Caravela), genótipo ABO-TEST (proveniente do assentamento Oito de Outubro, município de Simão Dias-SE), híbrido Samantha (proveniente da UFLA em MG), e o genótipo melhorado ABO-MELHOR (oriundo do Programa de Melhoramento de Plantas da UFS-DEA). Os tipos de fruto dos genótipos de abóbora estudados foram: Itapuã (globular achatado com gomos, casca verde acinzentada); Menina Rajada (cilíndrico com pescoço, casca verde rajada); Mini Paulista (cilíndrico com pescoço, casca verde com listras); Nova Caravela (oblongo, casca creme); ABO-TEST (cordiforme, casca alaranjada); ABO-MELHOR (cordiforme, casca alaranjada); Samantha (fruto achatado, casca verde escura).

Foi utilizado o delineamento experimental em blocos casualizados com sete tratamentos (genótipos) e três repetições, sendo as parcelas de $12 \times 12$ $\mathrm{m}$. Os espaçamentos utilizados foram 1x1 m para o híbrido, 2x2 m para a cultivar Mini Paulista e 3x3 $\mathrm{m}$ para os demais genótipos. As avaliações foram feitas utilizando a parcela útil de $6 \times 6$ $\mathrm{m}$, na parte central da parcela, onde foram colhidos e pesados os frutos. As avaliações foram efetuadas em cinco frutos obtidos aleatoriamente em cada parcela, que apresentavam características que mais se aproximavam das estabelecidas para cada cultivar. A parcela foi preparada realizando-se aração, gradagem e as sementes distribuídas por semeadura direta. As adubações foram feitas de acordo com a recomendação para a cultura da aboboreira, proposta por Filgueira (2008). Os tratos culturais adotados foram os recomendados para a cultura.

A colheita do primeiro ensaio foi realizada com aproximadamente 100 dias após o plantio, devido ao excesso de chuvas no período de maturação dos frutos. O segundo ensaio foi colhido aos 110 dias, quando os frutos apresentaram sinais visuais de maturação, como senescência do pedúnculo e mudança de coloração e brilho na casca.

A partir dos dados obtidos foi realizada a análise de variância de cada ano, para estimar a relação entre o maior e o menor quadrado médio do resíduo para cada ano. As variáveis foram submetidas à análise de variância conjunta, segundo modelo proposto por Vencovsky \& Barriga (1992), considerando como aleatório o efeito de anos e fixo o efeito de populações.

As estimativas dos parâmetros genéticos e fenotípicos, como a herdabilidade no sentido amplo $\left(\mathrm{h}^{2}\right)$, variância genética $\left(\mathrm{V}_{\mathrm{g}}\right)$, variância residual $\left(\mathrm{V}_{\mathrm{e}}\right)$, coeficiente de variação $(\mathrm{CV})$, variância genótipo $\mathrm{x}$ ambiente e relação $\mathrm{CV}_{\mathrm{g}} / \mathrm{CV}_{\mathrm{e}}$ foram efetuadas por meio do programa GENES (Aplicativo Computacional em Genética e Estatística) (Cruz, 2006).

Os dados foram submetidos à análise de variância e posteriormente ao teste de agrupamento das médias de Scott-Knott a 5\% de probabilidade com o auxílio do Programa SISVAR (Ferreira, 2008).

\section{RESULTADOS E DISCUSSÃO}

Diferenças significativas foram observadas na fonte de variação Anos para os caracteres largura do fruto, espessura da polpa na região mediana do fruto, espessura da polpa na região inferior do fruto, largura da cavidade interna do fruto, altura da cavidade interna do fruto e massa fresca de 100 sementes. Para a fonte de variação Genótipos observou-se diferenças significativas para todas as características. Para a interação Genótipos x Anos observou-se diferenças significativas para as características comprimento do fruto, largura do fruto, espessura da polpa na região mediana do fruto, espessura da polpa na região infe- 
Tabela 1. Parâmetros genéticos para comprimento do fruto (ALTF) (cm), largura do fruto (LARGF) (cm), espessura da polpa na região do pedúnculo $(\mathrm{EPP})(\mathrm{cm})$, espessura da polpa na região mediana do fruto $(\mathrm{EPM})(\mathrm{cm})$, espessura da polpa na região inferior do fruto $(\mathrm{EPI})(\mathrm{cm})$, largura da cavidade interna do fruto (LARGCV) $(\mathrm{cm})$, altura da cavidade interna do fruto (ALTCV) (cm), rendimento de fruto (RF) (cm), número de sementes por fruto (NSE) e massa fresca de 100 sementes (MSE) (g) de sete genótipos de abóbora (Cucurbita spp.) cultivados em dois anos (genetic parameters for fruit length (ALTF) $(\mathrm{cm})$, fruit width (LARGF) (cm), pulp thickness in the peduncle (EPP) (cm), pulp thickness in the middle region of the fruit $(\mathrm{EPM})(\mathrm{cm})$, pulp thickness in the lower region of the fruit $(\mathrm{EPI})(\mathrm{cm})$, width of the fruit internal cavity (LARGCV) $(\mathrm{cm})$, height of the fruit internal cavity (ALTCV) (cm), fruit yield (RF) (cm), number of seeds per fruit (NSE), fresh weight of 100 seed (MSE) (g) of seven pumpkin (Cucurbita spp.) genotypes cultivated over two years). São Cristóvão, UFS. 2010.

\begin{tabular}{|c|c|c|c|c|c|c|c|c|c|c|}
\hline \multirow{2}{*}{ Caracteres } & ALTF & LARGF & EPP & EPM & EPI & LARGCV & ALTCV & $\mathbf{R F}$ & NSE & MSE \\
\hline & \multicolumn{10}{|c|}{$C V_{g}(\%)$} \\
\hline 2008 & 12,74 & 5,75 & 25,10 & 15,41 & 8,27 & 21,85 & 16,50 & 10,54 & 16,50 & 10,54 \\
\hline \multirow[t]{2}{*}{2009} & 17,89 & 8,64 & 41,32 & 9,69 & 16,60 & 43,34 & 22,80 & 13,52 & 22,80 & 13,52 \\
\hline & \multicolumn{10}{|c|}{$h^{2}$} \\
\hline 2008 & 0,98 & 0,98 & 0,98 & 0,73 & 0,99 & 0,97 & 0,92 & 0,95 & 0,92 & 0,95 \\
\hline 2009 & 0,97 & 0,95 & 0,96 & 0,93 & 0,97 & 0,76 & 0,77 & 0,86 & 0,77 & 0,86 \\
\hline Variância genética $\left(\mathrm{V}_{\mathrm{g}}\right)$ & 261,45 & 13,11 & 120,62 & 1,16 & 0,00 & 2,59 & 81,95 & 19,47 & 14586,09 & 17,38 \\
\hline Variância residual $\left(\mathrm{V}_{\mathrm{e}}^{\mathrm{g}}\right)$ & 16,55 & 1,49 & 9,11 & 0,18 & 1,48 & 2,09 & 3,21 & 10,88 & 6741,35 & 8,51 \\
\hline $\begin{array}{l}\text { Variância da interação } \\
\text { genótipos x ambientes }\end{array}$ & 0,00 & 1,23 & 2,63 & 0,45 & 9,06 & 1,05 & 0,49 & 19,29 & 373,57 & 12,13 \\
\hline $\mathrm{h}^{2} \%$ & 99,81 & 93,13 & 97,52 & 79,66 & 0,00 & 72,97 & 99,00 & 59,84 & 91,57 & 67,16 \\
\hline $\mathrm{CV}_{\mathrm{g}}(\%)$ & 61,02 & 21,49 & 123,82 & 31,93 & 0,00 & 14,76 & 68,68 & 44,56 & 29,05 & 17,53 \\
\hline $\mathrm{CV}_{\mathrm{e}}(\%)$ & 15,35 & 7,25 & 34,03 & 12,76 & 29,89 & 13,25 & 13,60 & 33,32 & 19,75 & 12,27 \\
\hline
\end{tabular}

$\mathrm{CV}_{\mathrm{g}}(\%)$ : coeficiente de variação genética. $\mathrm{CV}_{\mathrm{e}}(\%)$ : coeficiente de variação ambiental $\left[\left(\mathrm{CV}_{\mathrm{g}}(\%)\right.\right.$ : genetic variance index. $\mathrm{CV}(\%)$ : environmental variance index)].

rior do fruto, largura da cavidade interna do fruto, rendimento de frutos e massa fresca de 100 sementes, demonstrando que houve influência dos anos sobre o comportamento dos genótipos. Dados similares foram obtidos por Espitia et al. (2006), ao avaliar sete híbridos de abóbora (C. moschata).

A espessura da polpa na região do pedúnculo foi o caráter que apresentou os maiores coeficientes de variação genética $\left(\mathrm{CV}_{\mathrm{g}}\right)(25,10$ e 41,32$)$ nos dois anos agrícolas respectivamente (Tabela 1). Estes valores estão acima dos observados por Bezerra Neto et al. (2006) ao estimar parâmetros genéticos em sete genótipos de C. moschata, obtendo o valor 9,33 para espessura da polpa.

Nos dois anos agrícolas avaliados, a herdabilidade foi acima de $90 \%$ para os caracteres comprimento do fruto $(98$ e $97 \%$ ), largura do fruto (98 e 95\%), espessura da polpa na região do pedúnculo (98 e 96\%), e espessura da polpa na região inferior do fruto ( 99 e $97 \%$ ) (Tabela 1). Ao avaliar 133 acessos de $C$. moschata na Colômbia, encontraram-se valores de herdabilidade abaixo dos observados neste estudo (Montes et al., 2006). Os altos valores observados para a herdabilidade indicam que os caracteres podem ser passados às gerações seguintes por seleção com grande chance de sucesso (Tabela 1).

No ano agrícola de 2009, os coeficientes de variação genética mostraram-se superiores em relação ao ano anterior, exceto para o caráter espessura da polpa na região mediana do fruto sendo a largura do fruto a variável que apresentou os menores valores para os dois anos $(5,75$ e $8,64 \%)$ (Tabela 1$)$.

A variância genética foi maior que a sua interação com o ambiente para quase todas as características, demonstrando que o ambiente pouco influiu na estimativa destas características (Tabela 1). Somente para espessura da polpa na região inferior do fruto, que apresentou variância genética nula, indicando maior influência do componente ambiente que os demais. Em seu estudo com sete linhagens de C. moschata, Bezerra Neto et al. (2006) obtiveram valor abaixo de 1,0 para este caráter $(0,08)$.

A interação genótipo $\mathrm{x}$ ambiente apresentou-se inferior à genética para todos os caracteres, exceto para o caráter espessura da polpa na região inferior do fruto (Tabela 1). Montes et al. (2006), avaliando 133 acessos de C. moschata na Colômbia, observaram uma alta influência do ambiente (considerando a diferença entre as variâncias fenotípicas e genotípicas), sendo os caracteres altura de planta e rendimento de frutos os mais afetados.

As estimativas dos coeficientes de herdabilidade apresentam as mesmas tendências observadas para os coeficientes de variação genética e suas magnitudes expressam a variabilidade genética exibida pelos genótipos. As estimativas de herdabilidade foram inferiores a $80 \%$ para os caracteres espessura da polpa na região inferior do fruto $(0,00 \%)$, largura da cavidade interna do fruto $(72,97 \%)$, rendimento de frutos $(59,84 \%)$ e massa fresca de 100 sementes $(67,16 \%)$. De acordo com Falconer (2003), quando os valores da herdabilidade $\left(\mathrm{h}^{2}\right)$ são superiores a $80 \%$, podem ser obtidos ganhos de seleção satisfatórios. Tal situação foi observada para os caracteres: comprimento e largura de fruto, espessura da polpa na região mediana do fruto, espessura da polpa na região do pedúnculo, altura da cavidade interna do fruto e número de sementes/fruto, indicando que genótipos superiores para 
Tabela 2. Análise conjunta para comparação das médias dos caracteres para comprimento do fruto (ALTF) (cm), largura do fruto (LARGF) $(\mathrm{cm})$, espessura da polpa na região do pedúnculo $(\mathrm{EPP})(\mathrm{cm})$, espessura da polpa na região mediana do fruto $(\mathrm{EPM})(\mathrm{cm})$, espessura da polpa na região inferior do fruto $(\mathrm{EPI})(\mathrm{cm})$, largura da cavidade interna do fruto (LARGCV) $(\mathrm{cm})$, altura da cavidade interna do fruto (ALTCV) $(\mathrm{cm})$, rendimento de frutos $(\mathrm{RF})(\mathrm{cm})$, número de sementes por fruto (NSE) e massa fresca de 100 sementes (MSE) (g) de sete genótipos de abóbora (Cucurbita spp.) cultivados em dois anos (joint analysis for comparison of means for fruit length (ALTF) (cm), fruit width $(\mathrm{LARGF})(\mathrm{cm})$, pulp thickness in the peduncle $(\mathrm{EPP})(\mathrm{cm})$, pulp thickness in the middle region of the fruit $(\mathrm{EPM})(\mathrm{cm})$, pulp thickness in the lower region of the fruit (EPI) $(\mathrm{cm})$, width of the fruit internal cavity (LARGCV) $(\mathrm{cm})$, height of the fruit internal cavity (ALTCV) (cm), fruit yield (RF) (cm), number of seeds per fruit (NSE), fresh weight of 100 seeds (MSE) (g) of seven pumpkin (Cucurbita spp.) genotypes cultivated over two years). São Cristóvão, UFS. 2010.

\begin{tabular}{|c|c|c|c|c|c|c|c|c|c|c|}
\hline \multirow{2}{*}{ Genótipo } & ALTF & LARGF & EPP & EPM & EPI & LARGCV & ALTCV & RF & NSE & MSE \\
\hline & \multicolumn{10}{|c|}{2008} \\
\hline Samantha & $12,30 \mathrm{c}$ & $20,34 \mathrm{a}$ & $2,42 \mathrm{c}$ & $4,06 \mathrm{~b}$ & $3,18 \mathrm{c}$ & $12,19 \mathrm{~b}$ & $6,76 \mathrm{c}$ & $24,80 \mathrm{a}$ & $187,00 \mathrm{c}$ & $32,05 \mathrm{a}$ \\
\hline Itapuã & $15,15 \mathrm{c}$ & $17,74 \mathrm{~b}$ & $3,39 \mathrm{c}$ & $3,16 \mathrm{c}$ & $2,66 \mathrm{c}$ & $10,95 \mathrm{~b}$ & $9,66 \mathrm{~b}$ & $7,00 \mathrm{c}$ & $502,66 \mathrm{a}$ & $26,23 \mathrm{~b}$ \\
\hline ABO-TEST & $19,53 \mathrm{~b}$ & $19,75 \mathrm{a}$ & $6,08 \mathrm{c}$ & $6,93 \mathrm{a}$ & $6,25 \mathrm{~b}$ & $12,30 \mathrm{~b}$ & $10,00 \mathrm{~b}$ & $6,44 \mathrm{c}$ & $548,00 \mathrm{a}$ & $25,87 \mathrm{~b}$ \\
\hline ABO-MELHOR & $19,90 \mathrm{~b}$ & $19,67 \mathrm{a}$ & $4,77 \mathrm{c}$ & $4,65 \mathrm{~b}$ & $4,67 \mathrm{~b}$ & $11,07 \mathrm{~b}$ & $10,50 \mathrm{~b}$ & $2,29 \mathrm{c}$ & $634,00 \mathrm{a}$ & $22,89 \mathrm{~b}$ \\
\hline Mini Paulista & $24,20 \mathrm{~b}$ & $8,44 \mathrm{c}$ & $1,69 \mathrm{c}$ & $1,62 \mathrm{~d}$ & $14,76 \mathrm{a}$ & $8,22 \mathrm{c}$ & $5,28 \mathrm{c}$ & $2,56 \mathrm{c}$ & $333,33 \mathrm{~b}$ & $11,75 \mathrm{~d}$ \\
\hline Nova Caravela & $47,20 \mathrm{a}$ & $17,47 \mathrm{~b}$ & $10,22 \mathrm{~b}$ & $4,13 \mathrm{~b}$ & $3,18 \mathrm{c}$ & $11,58 \mathrm{~b}$ & 32,19 a & $14,04 \mathrm{~b}$ & $378,33 \mathrm{~b}$ & $20,03 \mathrm{c}$ \\
\hline \multirow[t]{2}{*}{ Menina Rajada } & $54,04 \mathrm{a}$ & $18,22 \mathrm{~b}$ & $34,12 \mathrm{a}$ & $2,86 \mathrm{c}$ & $3,60 \mathrm{c}$ & $14,96 \mathrm{a}$ & $12,36 \mathrm{~b}$ & $15,69 \mathrm{~b}$ & $399,33 \mathrm{~b}$ & $20,07 \mathrm{c}$ \\
\hline & \multicolumn{10}{|c|}{2009} \\
\hline Samantha & $12,91 \mathrm{c}$ & $16,05 \mathrm{~b}$ & $1,99 \mathrm{c}$ & $2,39 \mathrm{~b}$ & $2,89 \mathrm{a}$ & $11,07 \mathrm{a}$ & $7,85 \mathrm{c}$ & $10,40 \mathrm{~b}$ & $224,33 \mathrm{c}$ & $28,88 \mathrm{a}$ \\
\hline Itapuã & & & $2,28 \mathrm{c}$ & $2,34 \mathrm{~b}$ & & $10,52 \mathrm{a}$ & $66 \mathrm{c}$ & $6,81 \mathrm{~b}$ & $404,00 \mathrm{~b}$ & $20,65 \mathrm{~b}$ \\
\hline ABO-TEST & $16,49 \mathrm{c}$ & $20,86 \mathrm{a}$ & $3,83 \mathrm{c}$ & $3,67 \mathrm{a}$ & $2,76 \mathrm{a}$ & $12,89 \mathrm{a}$ & $9,54 \mathrm{c}$ & $5,66 \mathrm{~b}$ & $573,33 \mathrm{a}$ & $25,88 \mathrm{a}$ \\
\hline ABO-MELHOR & $17,75 \mathrm{c}$ & $19,70 \mathrm{a}$ & $3,60 \mathrm{c}$ & $3,88 \mathrm{a}$ & $3,42 \mathrm{a}$ & $11,82 \mathrm{a}$ & $11,17 \mathrm{c}$ & $9,24 \mathrm{~b}$ & $503,33 \mathrm{a}$ & $24,25 \mathrm{a}$ \\
\hline Mini Paulista & $20,90 \mathrm{c}$ & $9,47 \mathrm{c}$ & $9,22 \mathrm{~b}$ & $1,68 \mathrm{~b}$ & $1,61 \mathrm{a}$ & $5,98 \mathrm{~b}$ & $10,08 \mathrm{c}$ & $4,45 \mathrm{~b}$ & 404,66 b & $15,47 \mathrm{c}$ \\
\hline Nova Caravela & $44,04 \mathrm{~b}$ & $16,30 \mathrm{c}$ & $7,40 \mathrm{~b}$ & $3,62 \mathrm{a}$ & $2,98 \mathrm{a}$ & $8,99 \mathrm{a}$ & $34,27 \mathrm{a}$ & $19,29 \mathrm{a}$ & 349,66 b & $30,73 \mathrm{a}$ \\
\hline \multirow[t]{2}{*}{ Menina Rajada } & $52,70 \mathrm{a}$ & $16,48 \mathrm{~b}$ & $33,11 \mathrm{a}$ & $2,30 \mathrm{~b}$ & $3,11 \mathrm{a}$ & $10,20 \mathrm{a}$ & $15,15 \mathrm{~b}$ & $9,92 \mathrm{~b}$ & $376,66 \mathrm{~b}$ & $28,14 \mathrm{a}$ \\
\hline & \multicolumn{10}{|c|}{ Média } \\
\hline Samantha & $0 \mathrm{~d}$ & $0 \mathrm{~b}$ & $2,20 \mathrm{c}$ & $3,22 \mathrm{c}$ & $3,03 \mathrm{c}$ & $3 \mathrm{a}$ & $30 \mathrm{~d}$ & $17,60 \mathrm{a}$ & $205,66 \mathrm{~d}$ & $30,46 \mathrm{a}$ \\
\hline Itapuã & $14,49 \mathrm{~d}$ & $16,50 \mathrm{~b}$ & $2,84 \mathrm{c}$ & $2,75 \mathrm{~d}$ & $2,30 \mathrm{c}$ & $10,73 \mathrm{a}$ & $9,66 \mathrm{c}$ & $6,91 \mathrm{c}$ & $453,33 \mathrm{~b}$ & $23,44 \mathrm{~b}$ \\
\hline ABO-TEST & $18,01 \mathrm{c}$ & $20,30 \mathrm{a}$ & $4,95 \mathrm{c}$ & $5,30 \mathrm{a}$ & $4,50 \mathrm{~b}$ & $12,59 \mathrm{a}$ & $9,77 \mathrm{c}$ & $6,05 \mathrm{c}$ & $560,66 \mathrm{a}$ & $25,87 \mathrm{~b}$ \\
\hline ABO-MELHOR & $18,82 \mathrm{c}$ & $19,69 \mathrm{a}$ & $4,18 \mathrm{c}$ & $4,26 \mathrm{~b}$ & $4,05 \mathrm{~b}$ & $11,45 \mathrm{a}$ & $10,83 \mathrm{c}$ & $5,76 \mathrm{c}$ & $568,66 \mathrm{a}$ & $23,57 \mathrm{~b}$ \\
\hline Mini Paulista & $22,55 \mathrm{c}$ & $8,96 \mathrm{c}$ & $5,46 \mathrm{c}$ & $1,65 \mathrm{e}$ & $8,19 \mathrm{a}$ & $7,10 \mathrm{~b}$ & $7,68 \mathrm{~d}$ & $3,50 \mathrm{c}$ & $369,00 \mathrm{c}$ & $13,61 \mathrm{c}$ \\
\hline Nova Caravela & $45,62 \mathrm{~b}$ & $16,88 \mathrm{~b}$ & $8,81 \mathrm{~b}$ & $3,87 \mathrm{~b}$ & $3,08 \mathrm{c}$ & $10,29 \mathrm{a}$ & $33,23 \mathrm{a}$ & $16,66 \mathrm{a}$ & $364,00 \mathrm{c}$ & $25,38 \mathrm{~b}$ \\
\hline Menina Rajada & $53,37 \mathrm{a}$ & $17,35 \mathrm{~b}$ & $33,61 \mathrm{a}$ & $2,58 \mathrm{~d}$ & $3,36 \mathrm{c}$ & $12,58 \mathrm{a}$ & $13,75 \mathrm{~b}$ & $12,80 \mathrm{~b}$ & $388,00 \mathrm{c}$ & $24,10 \mathrm{~b}$ \\
\hline $\mathrm{CV}(\%)$ & 15,35 & 7,26 & 34,03 & 12,76 & 22,83 & 13,26 & 13,60 & 33,32 & 19,76 & 12,27 \\
\hline
\end{tabular}

Médias seguidas pela mesma letra, nas colunas, não diferem entre si pelo teste de Scott-Knott ( $\mathrm{p} \leq 0,05)$ (means followed by the same letter in the columns did not differ from each other by the Scott-Knott test, $\mathrm{p} \leq 0.05$ ).

estes caracteres, podem ser obtidos pelo emprego de seleções simples (Tabela 1). Valores semelhantes também foram observados por Bezerra Neto et al. (2006), onde a herdabilidade foi de $91,99 \%$ para o caráter comprimento do fruto. Para a variação genética, apenas a variável espessura da polpa na região inferior do fruto apresentou variância genética nula (Tabela 1), indicando que não é uma variável a ser usada para seleção num programa de melhoramento.

O coeficiente de variância ambiental variou entre $7,25 \%$ para largura do fruto e $34,03 \%$ para espessura da polpa na região do pedúnculo, evidenciando uma menor complexidade no controle do primeiro caráter quando comparado com o segundo (Tabela 1). De acordo com Redig (2007), uma estratégia teórica que poderia ser empregada seria o uso de maior número de repetições.

Para a relação $\mathrm{CV}_{\mathrm{g}} / \mathrm{CV}_{\mathrm{e}}$ foram encontrados valores maiores que 1,0 para os caracteres comprimento e largura do fruto, espessura da polpa nas regiões do pedúnculo e mediana do fruto, largura e altura da cavidade interna do fruto, ren- dimento de frutos, número de sementes/ fruto e massa fresca de 100 sementes, sendo uma condição favorável à seleção das variáveis estudadas. Somente para a variável espessura da polpa na região inferior do fruto observou-se a relação $\mathrm{CV}_{\mathrm{g}} / \mathrm{CV}_{\mathrm{e}}$ nula (Tabela 1). A seleção é adequada para obtenção de ganhos quando a relação $\mathrm{CV}_{\mathrm{g}} / \mathrm{CV}_{\mathrm{e}}$ tende para valores maiores ou iguais a 1,0 (Vencovsky, 1987). Neste trabalho verificou-se perspectiva de ganhos genéticos para todas as características, exceto para o caráter espessura de polpa 
na região inferior do fruto.

Para o caráter comprimento do fruto, observou-se uma variação de $12,30 \mathrm{~cm}$ a 54,04 cm para os genótipos Samantha e Menina Rajada, respectivamente (Tabela 2). Os genótipos Nova Caravela e Menina Rajada são grandes, com formato alongado, e apresentaram os maiores valores de comprimento do fruto nos dois anos agrícolas. Os frutos dos genótipos Samantha e Itapuã, que possuem frutos com formato achatado, apresentaram os menores valores para o caráter exibindo em média $12,60 \mathrm{~cm}$ e 14,49 $\mathrm{cm}$ respectivamente. Observa-se na Tabela 2, que os valores para o comprimento dos frutos dos genótipos Itapuã, ABO-TEST, ABO-MELHOR, Mini Paulista, Nova Caravela e Menina Rajada, apresentaram valores menores no ano de 2009, em comparação ao ano de 2008. Barbosa (2009), avaliando a largura do fruto de nove genótipos de abóbora, verificou valores que variavam de $21,37 \mathrm{~cm}$ a $47,54 \mathrm{~cm}$ para os frutos maiores, e de 10,12 cm a 45,62 cm para os menores.

Houve diferença significativa entre os genótipos para o caráter largura de fruto na análise conjunta, sendo observado o menor coeficiente de variação (Tabela 2), em função de uma maior precisão experimental. Para o genótipo Mini Paulista, abóbora cilíndrica e com pescoço, observou-se os menores valores de largura de fruto nos dois anos agrícolas, 8,44 cm para 2008 e 9,47 cm para 2009 (Tabela 2). Nos dois anos agrícolas os genótipos ABO-TEST e ABO-MELHOR foram superiores para este caráter 19,75 e 19,67 cm respectivamente, para 2008; e 20,86 e $19,70 \mathrm{~cm}$ no ano de 2009. Resultados similares foram observados por Ramos (1996) trabalhando com 40 acessos de C. moschata.

No ano agrícola 2008 os valores para espessura da polpa na região do pedúnculo, variaram de $1,69 \mathrm{~cm}$ a $34,12 \mathrm{~cm}$ para os genótipos Mini Paulista e Menina Rajada, respectivamente (Tabela 2). Em 2009 esses valores variaram de 1,99 a $33,11 \mathrm{~cm}$ para os genótipos Samantha e Menina Rajada, respectivamente (Tabela 2). Os valores de espessura da polpa na região do pedúnculo foram coerentes com os encontrados por Bezerra Neto et al. (2006) e Barbosa (2009).

Houve diferenças entre os genótipos para espessura da polpa na região mediana do fruto. Em 2008, essa característica variou de 1,62 a $6,93 \mathrm{~cm}$ para os genótipos Mini Paulista e ABO-TEST respectivamente, enquanto em 2009 essa variação foi de 1,68 a $3,88 \mathrm{~cm}$ para os genótipos Mini Paulista e ABO-MELHOR (Tabela 2). Bezerra Neto et al. (2006) registraram valores que variaram de 2,32 a 3,71 $\mathrm{cm}$ ao avaliar as cultivares Jacarezinho e Caravela.

Para a espessura da polpa na região inferior do fruto, houve diferenças significativas entre os genótipos somente no ano agrícola de 2008, com variação de $2,66 \mathrm{~cm}$ a $14,76 \mathrm{~cm}$ para os genótipos Itapuã e Mini Paulista, respectivamente (Tabela 2). Em 2009 esses valores variaram de 1,61 a 3,42 cm para os genótipos Mini Paulista e ABO-MELHOR. Ramos et al. (1999), avaliando frutos de $C$. moschata, também no Nordeste, encontraram valores de espessura de polpa que variaram de 1,67 a 3,94 cm, sendo estes similares ao estudo apresentado.

Alguns genótipos destacaram-se em espessura da polpa devido ao tamanho comprido e à forma do pescoço do fruto, ocasionando maior variação nos caracteres de espessura da polpa na região do pedúnculo, com maior coeficiente de variação $(34,03 \%)$.

A espessura da polpa é característica importante em trabalhos de melhoramento de abóboras, pois frutos que apresentem polpa mais espessa conferem maior rendimento, fator importante para a comercialização e industrialização dos frutos, além de melhor aproveitamento ao serem descascados e transportados.

Para o caráter largura da cavidade interna do fruto, observaram-se diferenças significativas nos dois anos agrícolas, sendo que estes variaram de 8,22 a 14,96 cm para os genótipos Mini Paulista e Menina Rajada, respectivamente em 2008 e de 5,98 a 12,89 $\mathrm{cm}$ para os genótipos Mini Paulista e ABO-TEST em 2009 (Tabela 2). Para a altura da cavidade interna dos frutos das cultivares Nova Caravela e Samantha, observaram-se valores nos dois anos de avaliação, sendo 32,19 e 34,27 cm e 6,76 e 7,85 cm, respectivamente (Tabela 2). A obtenção de cultivares que possuam frutos com a cavidade interna pequena é um dos objetivos do melhoramento em Cucurbita (Ramos et al., 1999). A análise da cavidade interna é importante quando o melhoramento é feito visando a produção de sementes, pois os frutos que apresentam maior cavidade interna dispõem de maior espaço para produção destas.

Para o rendimento de frutos, os genótipos variaram nos dois anos agrícolas. No ano de 2008, para Samantha, observou-se a média de 24,80 $\mathrm{t} \mathrm{ha}^{-1} \mathrm{e}$ para o genótipo ABO-MELHOR 2,29 t ha $^{-1}$ (Tabela 2). A elevada produtividade de Samantha está associada ao seu vigor híbrido, em relação às cultivares de polinização aberta. Além disso, este genótipo requer um reduzido espaçamento (1x1 m) para cultivo, aumentando assim o número de plantas por área. No ano de 2009, para o genótipo Nova Caravela, obteve-se a mais alta média 19,29 $\mathrm{tha}^{-1}$ e para o genótipo Mini Paulista a mais baixa média 4,45 $\mathrm{t} \mathrm{ha}^{-1}$ (Tabela 2), não diferindo estatisticamente dos demais genótipos. A boa produtividade do genótipo Nova Caravela é decorrente da elevada massa dos frutos, que têm tamanhos grandes. Para o genótipo Mini Paulista, obteve-se menor média dos anos (3,50 tha $\left.{ }^{-1}\right)$ (Tabela 2), devido aos seus frutos que são de tamanho pequeno e com massa reduzida. Os genótipos ABO-TEST e ABO-MELHOR (média de 6,05 e 5,76, respectivamente) (Tabela 2), provavelmente não expressaram seus valores reais devido às condições ambientais que foram submetidos, pois os experimentos foram conduzidos fora de sua região de adaptação e em períodos com excesso e escassez de chuvas em fases importantes do desenvolvimento das plantas, o que comprometeu o rendimento dos frutos.

A variação observada para o caráter número de sementes por fruto foi de 187, para Samantha, a 634, para ABO-MELHOR, no ano 2008 (Tabela 2); e de 224 a 503, no ano 2009. Em frutos da cv. Piramoita, após sucessivas gerações de autofecundação, o número de sementes por fruto variou de 132 a 242 (Cardoso, 2004).

Para massa fresca de 100 sementes, houve diferenças estatísticas nos dois anos agrícolas, e os valores variaram 
de 11,75 para o genótipo Mini Paulista a 32,05 para Samantha no ano 2008, e de 15,47 para Mini Paulista a 30,73 para Nova Caravela, em 2009 (Tabela 2). Estes valores estão acima dos alcançados por Cardoso (2004) em frutos da cv. Piramoita, após sucessivas gerações de autofecundação. Já Barbosa (2009) obteve valores que se aproximaram aos dados apresentados neste estudo.

Todos os caracteres avaliados apresentaram alta herdabilidade no sentido amplo, o que indica a grande possibilidade deles serem transmitidos para as gerações futuras, exceto para o caráter espessura da polpa na região inferior do fruto que obteve herdabilidade nula. $\mathrm{Na}$ análise conjunta de médias, os genótipos tiveram comportamento diverso em relação aos caracteres avaliados.

\section{AGRADECIMENTOS}

Os autores agradecem ao Prof. Dr. Wilson R Maluf, pela concessão das sementes do híbrido Samantha, e à FAPITEC/SE, ao CNPq e ao ETENE/ FUNDECI/BNB, pelo financiamento da pesquisa.

\section{REFERÊNCIAS}

ASSIS JGA; RAMOS NETO DC; ROMÃO RL; FERREIRA MAJF. 2006. Diagnóstico de produção de abóboras nos Estados de Sergipe e Alagoas. Magistra 18: 69.
BARBOSA GS. 2009. Desempenho agronômico, caracterização morfológica e polínica de linhagens de abóbora (Cucurbita moschata) com potencial para o lançamento de cultivares. Campos dos Goytacazes: UENF. 92p. (Tese mestrado).

BASSO KC; RESENDE RMS; VALLE CB; GONÇALVES MC; LEMPP B. 2009. Avaliação de acessos de Brachiaria brizantha Stapf e estimativas de parâmetros genéticos para caracteres agronômicos. Acta Scientarum - Agronomy 31: 17-22.

BEZERRA NETO FV; LEAL NR; COSTA FR; GONÇALVES GM; AMARAL JÚNIOR AT; VASCONCELLOS HO; MELLO M. 2006. Análise biométrica de linhagens de abóbora. Horticultura Brasileira 24: 378-380.

CARDOSO AII. Depression by inbreeding after four sucessive self-pollination squash generations. 2004. Scientia Agrícola 61: 224-227.

CRUZ CD; REGAZZI AJ. 2001. Modelos biométricos aplicados ao melhoramento genético. Viçosa: UFV. 390p.

CRUZ CD. 2005. Princípios da Genética Quantitativa. Viçosa: UFV. 394p.

CRUZ CD. 2006. Programa Genes: aplicativo computacional em genética e estatística. Viçosa: UFV. 648p.

ESPITIA MM; VALLEJO FA; ARAMÉNDIZ H. 2006. Evaluacion agronomica de siete híbridos experimentales $\mathrm{F}_{1}$ de zapallo (Cucurbita moschata Duch. Ex Poir). Temas Agrarios 11: $32-42$.

FALCONER DS. Introdução à genética quantitativa. 1987. Viçosa: UFV. 279p.

FERREIRA DF. 2008. SISVAR: um programa para análises e ensino de estatística. Revista Symposium 6: 36-41.

FILGUEIRA FAR. 2008. Novo manual de olericultura: agrotecnologia moderna na produção e comercialização de hortaliças. Viçosa: UFV. 421p.

LUENGO RFA; PARMAGNANI RMN;
PARENTE MR; LIMA MFBF. 2000. Tabela de composição nutricional das hortaliças. Brasília: Embrapa Hortaliças. 4p. (Documentos, 26).

MONTES RC; VALLEJO CFA; BAENA GD. 2004. Diversidad genética de germoplasma colombiano de zapallo (Cucurbita moschata Dúchesne Exp. Prior) Acta Agronómica 53: 43-50.

RAMOS SRR. 1996. Avaliação da variabilidade morfoagronômica de abóbora (Cucurbita moschata Duch.) do Nordeste Brasileiro. Viçosa: UFV. 71p. (Tese mestrado).

RAMOS SRR; QUEIROZ MA; CASALI VWD; CRUZ CD. 1999. Recursos genéticos de Cucurbita moschata: caracterização morfológica de populações locais coletadas no Nordeste Brasileiro. In: QUEIROZ MA; GOEDERT CO; RAMOS SRR. Recursos genéticos e melhoramento de plantas para o Nordeste Brasileiro. Disponível em: http:// www.cpatsa.embrapa.br/catalogo/livrorg/ abobora.pdf

REDIG MSF. 2007. Estimativas de parâmetros genéticos em clones de priprioca (Cyperus sp.). Belém:UFRA. 63p. (Tese mestrado).

RUIZ E; SIGARROAA; CRUZ JA. 2004. Analisis dialelico del rendimiento y sus principales componentes em variedades de calabaza (Cucurbita moschata Duch) I. tabla dialelica de griffing. Revista Biologia 18: 65-73.

SILVA DB; WETZEL MV; FERREIRA MAJF; LOPES JF; BUSTAMANTE PG. 2006. Conservação de germoplasma de Cucurbita spp. a longo prazo no Brasil. Brasília: Embrapa Recursos Genéticos e Biotecnologia. 12p. (Documentos, 135).

VENCOVSKY R. 1987. Herança Quantitativa. In: PARTENIANI E; VIÉGAS GP. Melhoramento e produção de milho. Campinas: Fundação Cargill. 5:137-214.

VENCOVSKY R; BARRIGA P. 1992. Genética biométrica aplicada no fitomelhoramento. Ribeirão Preto: SBG. 496p. 